\title{
Intraventricular Pilocytic Astrocytoma in an Adult Patient
}

\author{
Joshua Hendrix and Zhenggang Xiong*
}

Department of Pathology and Laboratory Medicine, Robert Wood Johnson Medical School, Rutgers University, USA

*Corresponding author: Zhenggang Xiong, MD, PhD, Division of Neuropathology, Department of Pathology and Laboratory Medicine, Robert Wood Johnson Medical School and University Hospital, Rutgers University, 125 Paterson Street, MEB 231, New Brunswick, NJ 08903, USA, Tel: 732-235-7471

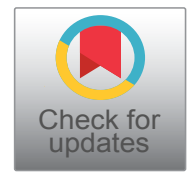

\begin{abstract}
Pilocytic astrocytomas are tumors of the central nervous system mostly during the first two decades of life. Although they are mostly common in the midline structures of children, pilocytic astrocytoma within the ventricular system of an adult is rare. To our knowledge, only one single histologically and molecularly confirmed case was documented in the literature up to this time. We report a case of a 38-yearold woman with obstructive hydrocephalus secondary to a brain tumor within the third ventricle. On histological examination, the tumor exhibited biphasic growth pattern comprising compacted cellular areas with Rosenthal fibers and loose textured microcystic areas with eosinophilic granular bodies. Mitosis or necrosis was not present. Immunohistochemical studies demonstrated glial fibrillary acid protein (GFAP) and Olig2 positivity as well as NeuN and EMA negativity. Ki67 labeling index was less than 1\%. Molecular studies revealed that there is no isocitrate dehydrogenase (IDH) gene mutation. This clinical presentation along with the histologic and molecular findings is consistent with a pilocytic astrocytoma arising in the third ventricle of this adult brain, which further supports that pilocytic astrocytoma can present as an intraventricular tumor in an adult patient and should be routinely included in the differential diagnosis of intraventricular neoplasm.
\end{abstract}

\section{Keywords}

Pilocytic astrocytoma, Ventricle, Central nervous system, Adult

\section{Introduction}

Pilocytic astrocytomas are tumors of the central nervous system (CNS) most commonly occurring during the first two decades of life with a peak incidence between 8 and 13 years of age. Although these tumors are typically located in the midline structures of CNS, like posterior fossa, cerebellum, thalamus, hypothala- mus, etc., they rarely arise within a cerebral ventricle of an adult patient [1]. Genetically, pilocytic astrocytomas are associated with absence of isocitrate dehydrogenase (IDH) gene mutation, and in most of cases, but not all cases, genomic changes in the mitogen-activated protein kinase (MAPK) pathway, particularly KIAA 1549/BRAF gene fusion, are present. Literature review indicates that only a single prior case of intraventricular pilocytic astrocytoma has been histologically and molecularly identified in an adult patient [2]. We report another histologically and molecularly confirmed case here to further support that pilocytic astrocytoma can occur intraventricularly in an adult patient.

\section{Case Description}

\section{Clinical information}

The patient was a 38-year-old woman with no significant medical history who reported a new brain mass and obstructive hydrocephalus recently diagnosed in another hospital. She presented to our institution for further evaluation and treatment. She described five months of intermittent diplopia, tremors, and clumsiness. She was previously treated with levetiracetam and acetazolamide. At our institution, computed tomography (CT) confirmed moderate hydrocephalus with dilation of the lateral and third ventricles, without dilation of the fourth ventricle, as well as transependymal resorption of CSF indicating acute hydrocephalus. Magnetic resonance imaging (MRI) revealed a $12 \mathrm{~mm}$ rounded mass along the floor of the third ventricle with mild to moderate heterogeneous contrast-enhancement, obstructing the cerebral aqueduct (Figure 1). Subsequently, she underwent endoscopic ventriculos- 


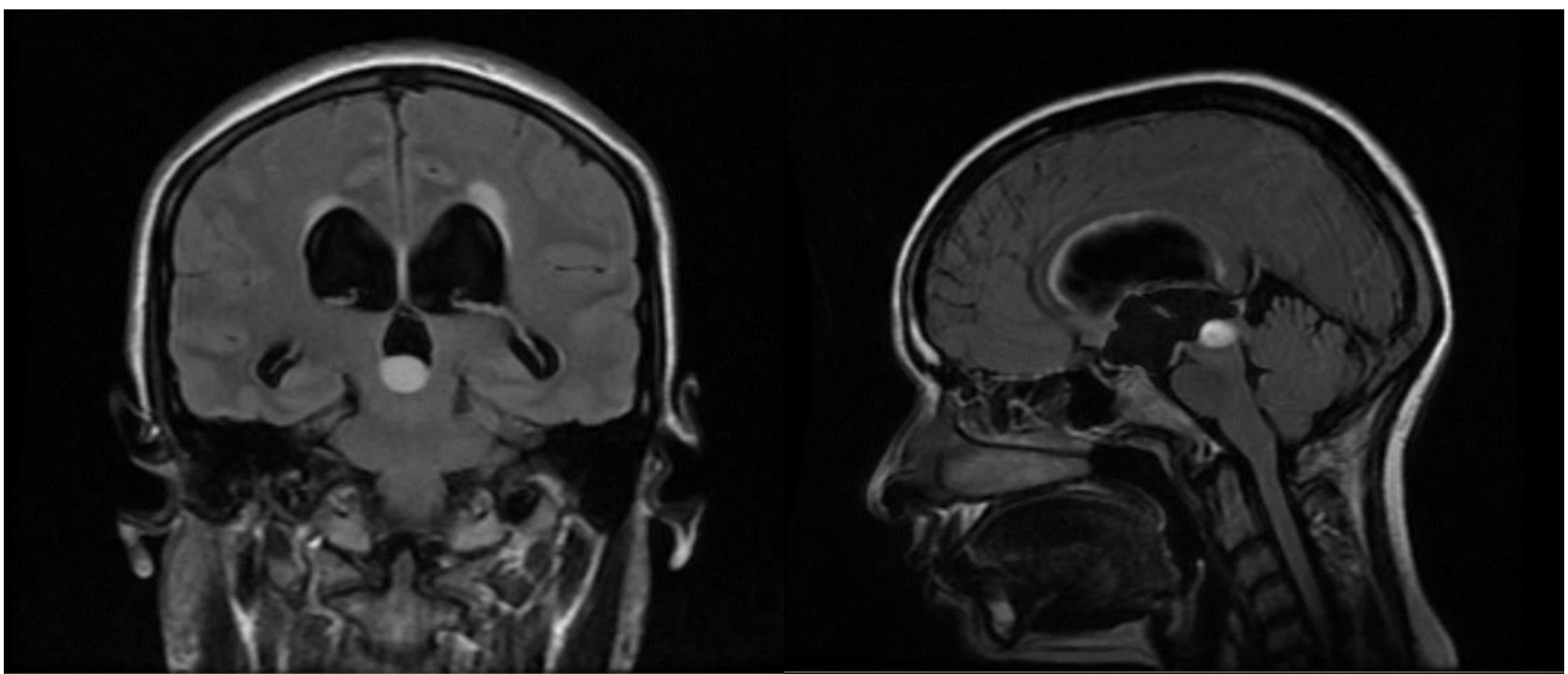

Figure 1: Magnetic Resonance Imaging, T2-weighted, showed a mild to moderate heterogeneous contrast-enhanced mass in the third ventricle.

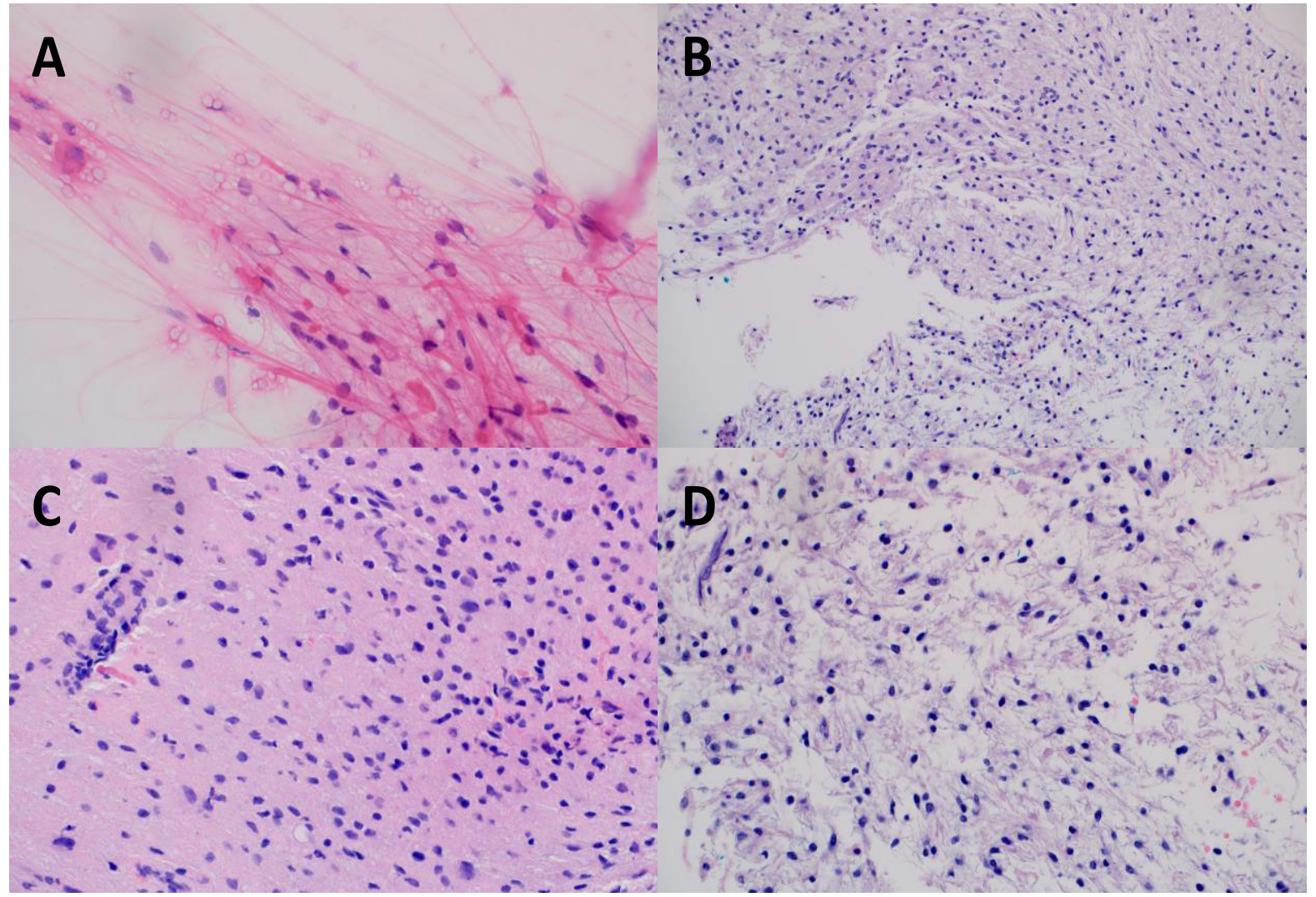

Figure 2: Histological examination observed two cell populations, bipolar piloid cells with long, hire-like processes and smaller cells with short, cobweb-like processes as well as a biphasic growth pattern composed of compacted cellular areas with Rosenthal fibers and loose textured microcystic areas with eosinophilic granular bodies. A) smear preparation, H\&E, 400x; B) tissue section, H\&E, 200x; C)-D) tissue section, H\&E, 400x.

tomy and excisional biopsy, in which a third ventricular tumor was identified and excised. The resulting specimen was sent to our neuropathology laboratory for evaluation.

\section{Pathology}

The surgical specimen comprised multiple small fragments of soft, pink-tan tissue. Cytological preparation for intra-operative consultation exhibited bipolar piloid cells with long, hair-like processes and elongated, moderately pleomorphic nuclei as well as smaller cells with short, cobweb-like processes and round to oval nuclei. Rosenthal fibers and eosinophilic granular bodies were present. Mitosis was not identified. Histological examination of the paraffin-embedded tissue revealed a biphasic growth pattern composed of compacted cellular 
areas with Rosenthal fibers and loose textured microcystic areas with eosinophilic granular bodies. Mitosis, vascular endothelial hyperplasia or necrosis was not observed (Figure 2). Immunohistochemical studies demonstrated that the neoplastic cells expressed glial fibrillary acid protein (GFAP) and Olig2, while IDH1 R132H, NeuN, and epithelial membrane antigen (EMA) were not present in the neoplastic cells. Ki67 labeling index was less than 1\% (Figure 3). Next-generation sequencing study showed that there was no IDH mutation, BRAF alterations including KIAA1549/BRAF fusion, and any other reported glioma-related genomic abnormalities (data not shown).

\section{Discussion}

An intraventricular tumor is far more likely to arise from choroid plexus, ependyma, and subependymal tissue. Other less common intraventricular tumors include meningioma, lymphoma, and metastasis. Third ventricle tumors are rare and only comprise $0.6-0.9 \%$ of all brain tumors among all age groups $[3,4]$. Within the category of third ventricle tumors, the most common includes colloid cysts, choroid plexus papillomas, and ependymomas. Occasionally, a pineal tumor can be located within the third ventricle. Although intraventricular pilocytic astrocytomas have been reported radiologically with or without limited histological examination $[5,6]$, only one histologically and molecularly confirmed pilocytic astrocytoma within the lateral ventricle has been recently documented in the literature [2]. In our case, neuroimaging demonstrated an obvious obstruct- ing third ventricle mass which histologically exhibited classic pilocytic astrocytoma features, including biphasic growth pattern comprising compacted cellular areas and loose textured microcystic areas with Rosenthal fibers and eosinophilic granular bodies. Immunohistochemical studies demonstrated that the tumor cells are positive for glial fibrillary acid protein (GFAP) and Olig2 with Ki67 labeling index of less than 1\%, indicating glial nature of low-grade neoplasm. Molecular studies revealed that there is no isocitrate dehydrogenase (IDH) gene mutation. The above supports the diagnosis of pilocytic astrocytoma. This case further supports that pilocytic astrocytoma can occur in the cerebral ventricular system including the third ventricle and should be in the differential diagnosis of intraventricular tumors.

Pilocytic astrocytoma accounts for $1.3 \%$ of all central nervous system tumors. It is the most common glioma in the pediatric population during the first two decades of life with median age of 12-yearsold, which declines dramatically from 14-years-old to 15-19 years old. Approximately one third of gliomas in 0-14 years old patients are pilocytic astrocytoma $[1,7]$. In adults, pilocytic astrocytoma is much less common. It usually occurs a decade earlier than diffuse astrocytoma and is rarely present in patients older than 50 years. It is worth noting that in contrast to another previously reported intrventricular pilocytic astrocytoma of an adult [2], this case does not harbor KIAA 1549/BRAF gene fusion. However, KIAA 1549/BRAF gene fusion is known to present much less frequently in adult patients with pilocyt-

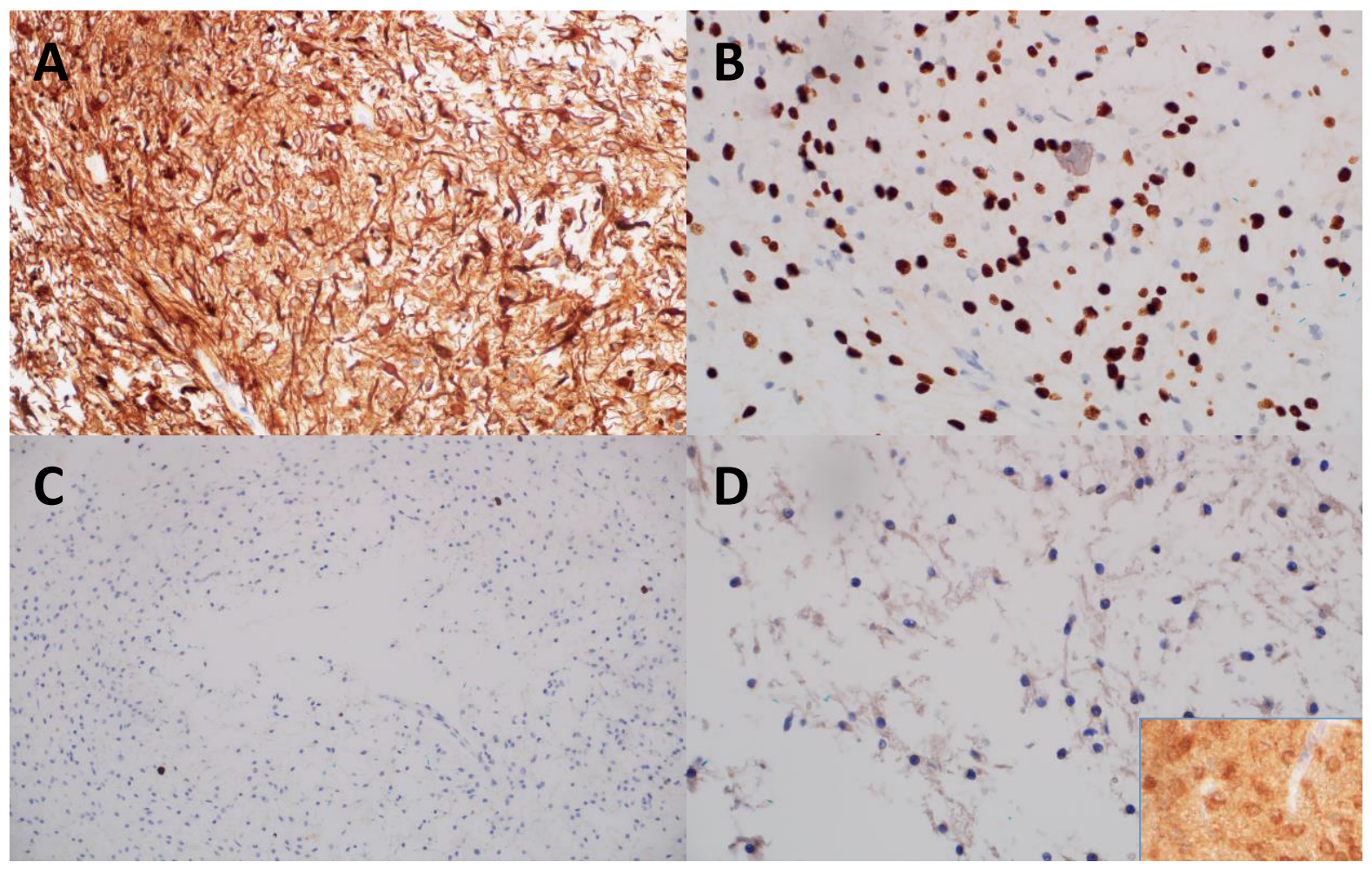

Figure 3: Immunohistochemical studies demonstrated that the neoplastic cells expressed GFAP and Olig2 with low Ki67 labeling index, but not IDH1 R132H mutant protein. A) GFAP immunostaining, 400x; B) Olig2 immunostaining, 400x; C) Ki67 immunostaining, 200x; D) IDH1 R132H immunostaining, 400x, insert: Positive control. 
ic astrocytoma, which suggests that the pathogenesis of intraventricular pilocytic astrocytoma may not necessarily be different from other extraventricular pilocytic astrocytomas [8].

\section{Conflict of Interest Statement}

The authors of this paper have no conflicts of interest to disclose. This paper was composed without funding from any institution.

\section{References}

1. Louis DN, Ohgaki H, Wiestler OD, Cavenee WK, Ellison DW, et al. (2016) WHO Classification of Tumours of the Central Nervous System. (4th edn), IARC Publications, Lyons.

2. Ahn JS, Harrison W, Hughes E, McLendon RE (2019) Intraventricular pilocytic astrocytoma with KIAA1549/BRAF fusion arising in a 44-year old. J Neuropathol Exp Neurol 78: $187-190$.
3. Ahmed SI, Javed G, Laghari AA, Bareeqa SB, Aziz K, et al. (2018) Third ventricular tumors: A comprehensive literature review. Cureus 10: e3417.

4. Glastonbury CM, Osborn AG, Salzman KL (2011) Masses and malformations of the third ventricle: Normal anatomic relationships and differential diagnoses. Radiographics 31: 1889-1905.

5. Xia JG, Yin B, Liu L, Lu YP, Geng DY, et al. (2016) Imaging features of pilocytic astrocytoma in cerebral ventricles. ClinNeuroradiol 26: 341-346.

6. Smith AB, Smirniotopoulos JG, Horkanyne-Szakaly I (2013) Intraventricular neoplasms: Radiologic-pathologic correlation. Radiographics 33: 21-43.

7. Ostrom QT, Cioffi G, Gittleman H, Patil N, Waite K, et al. (2019) CBTRUS Statistical Report: Primary brain and other central nervous system tumors diagnosed in the United States in 2012-2016. Neuro Oncol 21: v1-v100.

8. Collins VP, Jones DT, Giannini C (2015) Pilocytic astrocytoma: pathology, molecular mechanisms and markers. Acta Neuropathol 129: 775-788. 